\title{
THE PROTECTIVE PROPERTIES OF NEW AGONISTS OF FPR2 ON THE EVOKED BY BACTERIAL ENDOTOXIN CHANGES IN MICROGLIA CELLS
}

Agnieszka Basta-Kaim ${ }^{1}$, Monika Leśkiewicz¹, Magdalena Regulska1 , Ewa Trojan¹, Enza Lacivita², Marcello Leopoldo²

1 Department of Experimental Neuroendocrinology, Maj Institute of Pharmacology PAS, Cracow 12 Smętna Str, PL 31-343 Cracow, Poland,

2 Department of Pharmacy - Drug Sciences; University of Bari, via Orabona 4, 70125 Bari, Italy

\section{Introduction}

Neuroinflammation is a complex multicellular process that plays an important role in the onset and progression of neurodegenerative disorders, including Alzheimer's disease (AD). It is believed that a short-term inflammation, as a beneficial phenomenon, guarantees repair and restoration of homeostasis, whereas failure in resolution of inflammation (RoI) results in long-lasting, chronic inflammation. Recently, it has been shown that RoI is regulated by endogenous molecules, called specialized pro-resolving mediators (SPMs) like lipoxins, resolvins, protectins and others. SPMs modulate RoI by interacting with specific membrane receptor, such as Formyl peptide receptor 2 (FPR2).

\section{Aim of the study}

The aim of this study was to determine, whether specialized pro-resolving mediators (SPMs) such as endogenous lipoxin A4 (LXA $\mathbf{L A}_{4}$, its analog - aspirin triggered lipoxin (AT-LXA) as well as new non-peptidic agonists with ureidopropanamide scaffold (MR-39 and AMS) are able to affect the cell death processes evoked by lipopolysaccharide (LPS) treatment in primary microglia cells.

Fig. 3. New non-peptidic agonist - MR-39
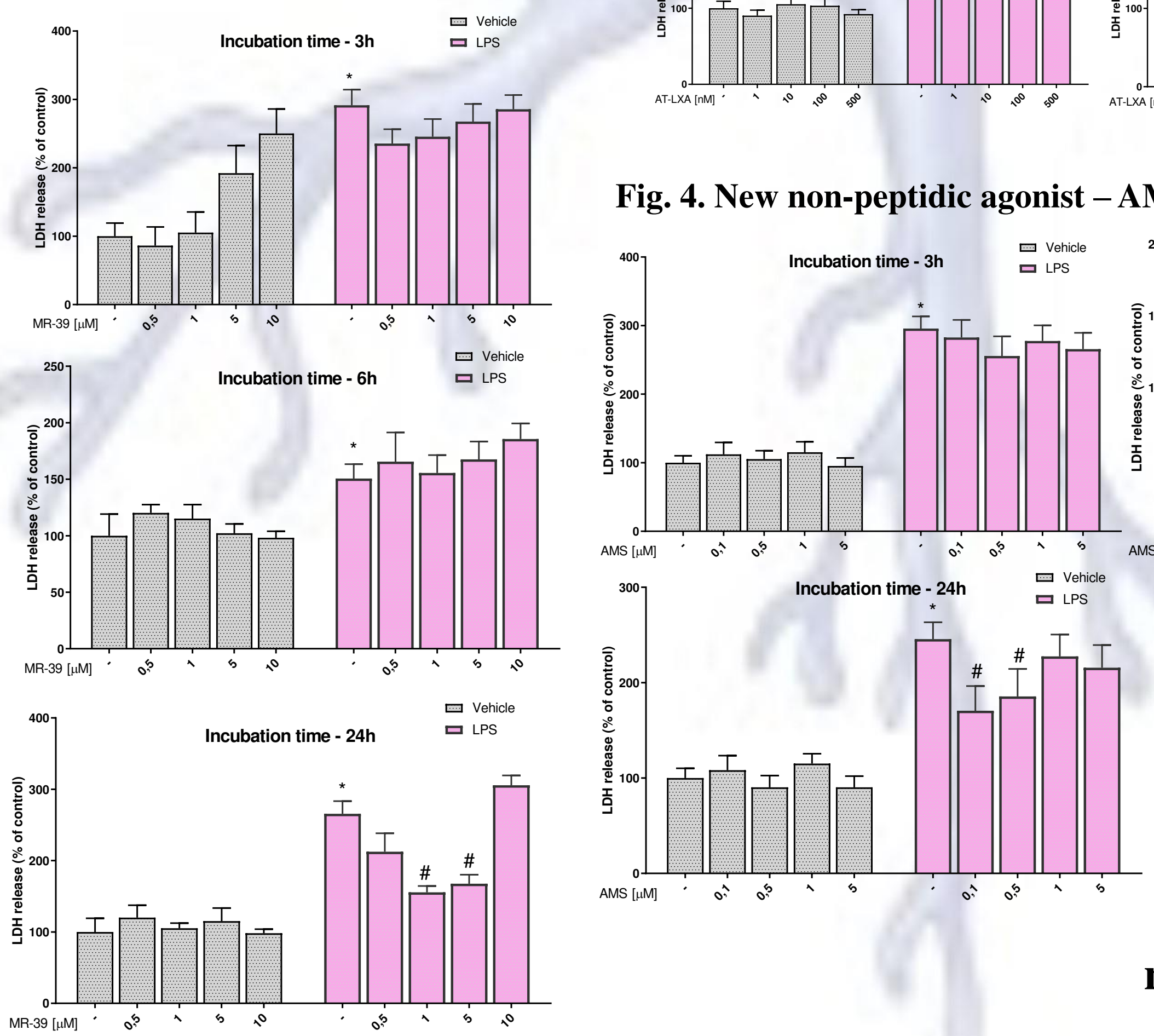

Fig. 1. Lipoxin A4

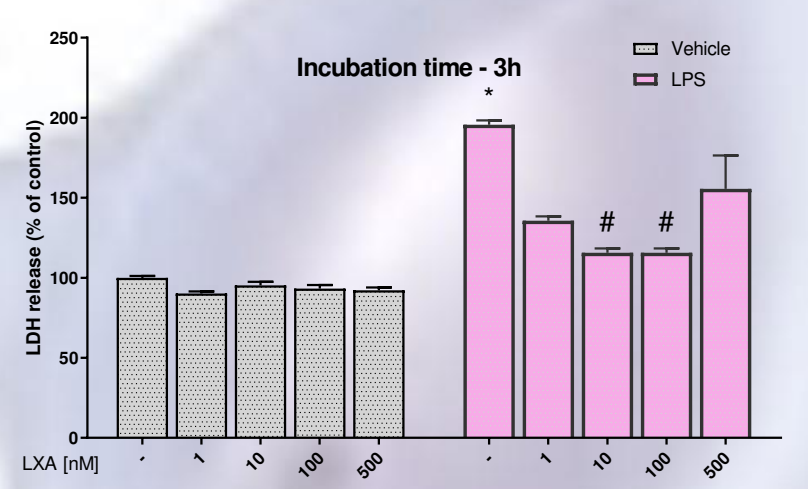

\section{Methods}

Female Sprague-Dawley rats were paired with males. Pregnancy was confirmed the next morning. Primary microglia cultures were prepared from cortices of 1-2 day old offspring. Microglia cells were pretreated with each compound (1-500 nM) and next exposed to nonspecific immune system activator - LPS in the concentration of 100 $\mathrm{ng} / \mathrm{ml}$. Time-dependent (3-24 h) cell death was determined by lactate dehydrogenase release (LDH test).

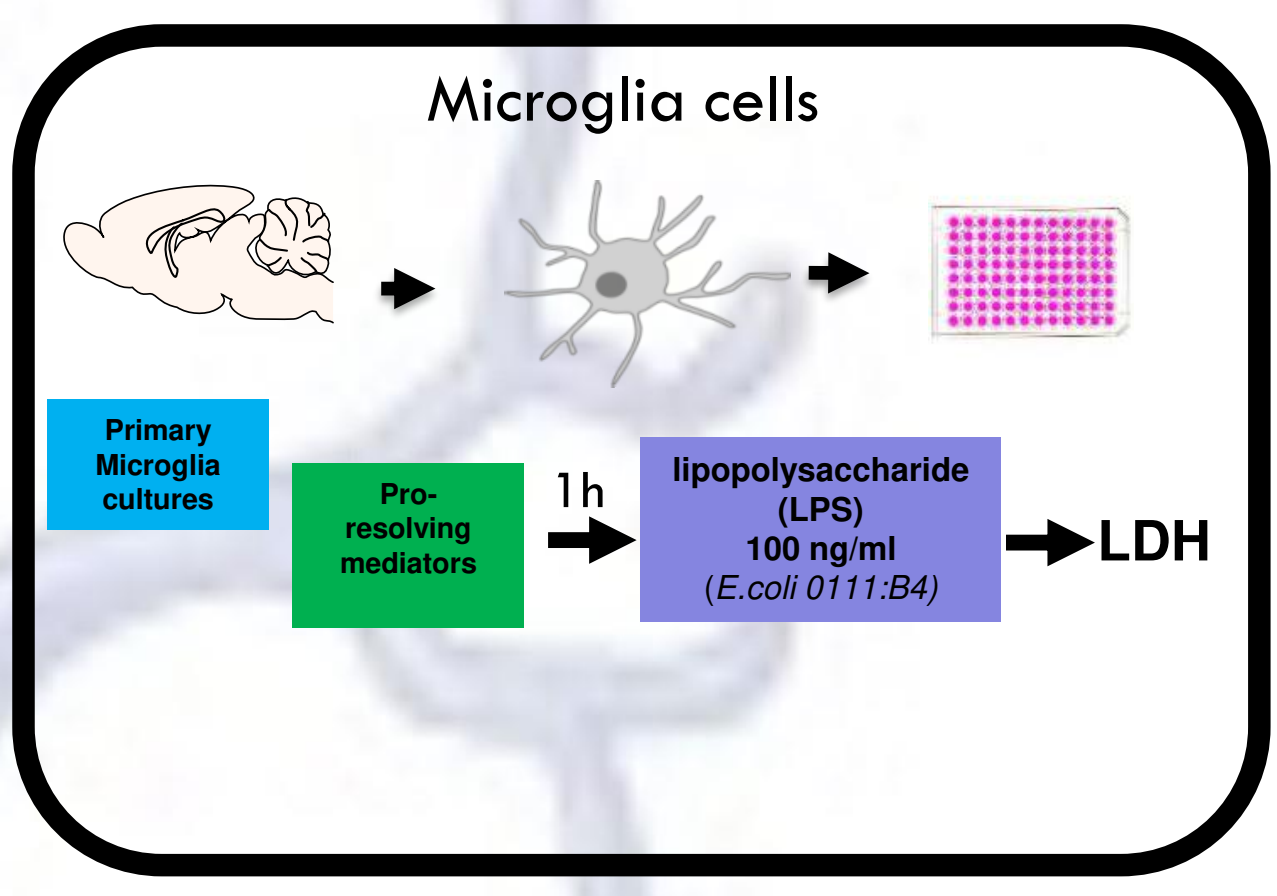

\section{Results}

Fig. 2. Aspirin triggered lipoxin
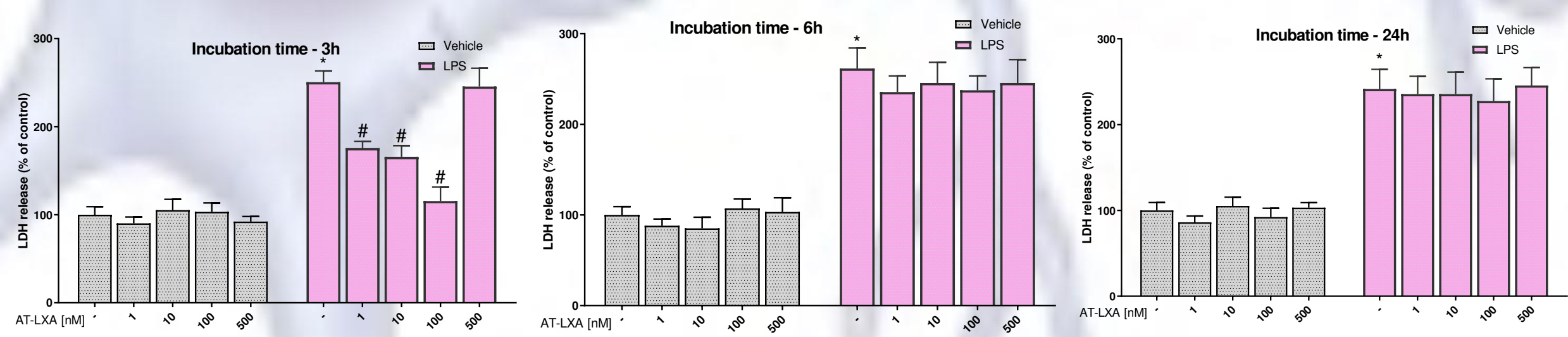

Fig. 4. New non-peptidic agonist - AMS

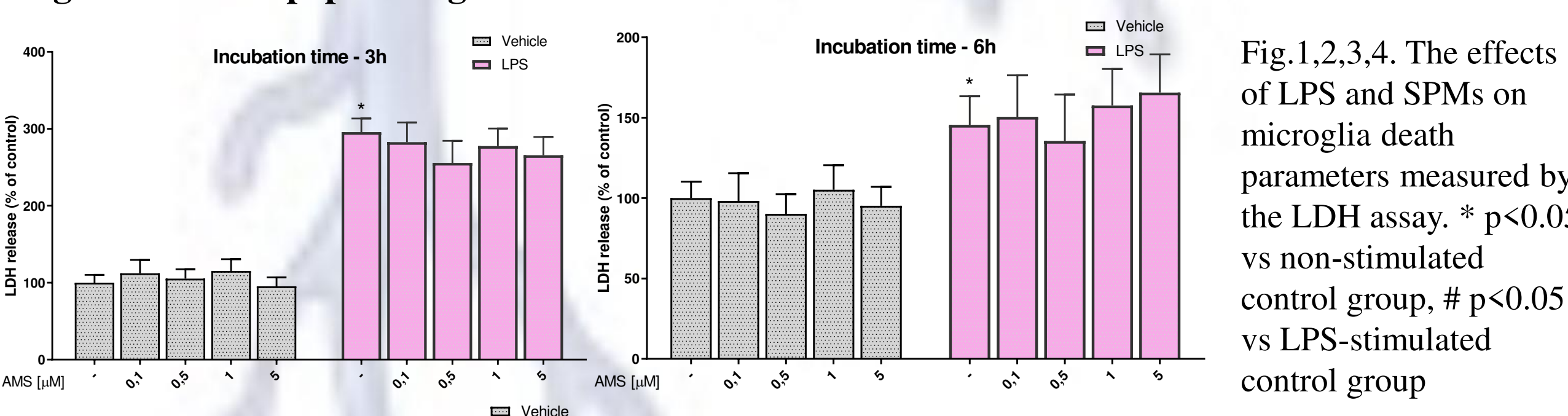

\section{Conclusions}

Our results expanded our current understanding of the role of FPR2 in neuroprotection. Moreover, the agonists of FPR2 can be consider as an attractive target for protective therapies. 